\title{
COVID 19 - Coronavirus disease Pandemic India
}

\author{
MANISHA YADAV \\ Mini.mba2006@gmail.com
}

\begin{abstract}
The Covid 19 coronavirus disease had been observed in Wuhan province of China, Which is now spreading all over the world. This disease is caused by severe acute respiratory syndrome (SARS).
\end{abstract}

We will cover the basics about this disease such as its symptoms, preventive measures and treatments. Although it will be very vast area of research many questions arises which requires answers, still we hope that this review helps in understanding this disease.

Keywords - Introduction, Symptoms, Prevention, Treatment, Myth and Facts about Covid - 19.

\section{INTRODUCTION}

The Novel coronavirus was named Coronavirus disease 2019 (Covid - 19) by World Health Organization (WHO) in February 2020. This virus also known as SARS - Cov 2 and the associated disease is COVID - 19.Coronaviruses is a family of viruses that cause illness such as respiratory disease. Firstly this disease was identified in Wuhan China and resulted in an ongoing Pandemic. Other names given to Covid - 19 are Coronavirus, Corona, COVID-19-n cov acute respiratory disease, Novel Coronavirus pneumonia, and severe pneumonia with novel pathogens.

\section{SyMPTOMS}

This disease (COVID - 19) affects different people in a different manner. Mostly infected persons will develop to moderate illness and can make a quick recovery without hospitalization. Most common symptoms are - fever, dry cough, tiredness, sore throat, headache, loss of taste or smell, rashes on a skin.

\section{Prevention}

We all have heard that prevention is always better than cure so we all have to protect ourselves and taking appropriate precautions. To prevent the spread of COVID -19 we should-

a. Clean our hands very often with soap and waters or an alcohol based sanitizers.

b. Maintaining a social distance (at least 1 meter) from others who is coughing or sneezing.

c. Do not frequently touch your nose, eyes or mouth.

d. Cover your face with mask while going outside to buy essentials.

e. If you feel unwell then stay home.

f. If you have a fever, cough, difficulty in breathing, seek medical advice.

g. Avoid going to crowded places.

h. Avoid shaking hands.

\section{TreatMentS}

Till date there are no specific vaccines or medicines are found to deal with COVID - 19. Investigations are going on to find out suitable vaccine.

Self-care is very important treatment. All of us should drink plenty of fluids which include warm water and must eat nutritious food. Stay active, make yoga and exercise a part of life, make social contact through phone or internet. Children's needs extra attention from parents during difficult times. If you feel sick or have any mild symptoms then you should self - isolate and seek medical attention.

\section{MYTh AND FACTS ABOUT COVID - 19}

It is truly said that in the absence of information we can jump to the worst conclusion. So it is always good to have a full information about something, therefore knowing the facts about Covid-19 symptoms can help to protect you and yours community too.

Myth 1 - The person get Covid -19 feels very sick and die?

Fact - No, Most people who get Covid-19 will have a mild form of illness and can recover without needing professional medical care. Around 8 out of 10 people will have mild symptoms. Around one in six people will seek hospital care. Scientific modeling suggests that around 1 in 100 people who get Covid-19- will die.

Myth 2- Does Covid-19 affects older people more rather than young people?

Fact - No, people of all ages can be infected by Covid-19. Older people with pre-existing medical condition such as asthma, diabetes, heart disease seems to be more affected by virus.

Myth 3- Can eating garlic helps prevent infection from Covid-19? 
Fact - Garlic have some antimicrobial properties and a healthy food. But there is no such evidence that it can prevent infection from Covid-19.

Myth 4- The person who can hold its breath for 10 seconds or more without feeling discomfort is free from Covid-19 disease?

Fact - No, you cannot confirm it with this breathing exercise, which can even be dangerous. The most common symptoms are dry cough, tiredness and fever.

Myth 5- Does the sunshine kills the coronavirus?

Fact - You can catch Covid-19, no matter how hot the weather is. Countries with hot weather have report cases of Covid-19.

\section{REFERENCES}

1. https://www.who.int

2. https://www/avert.org

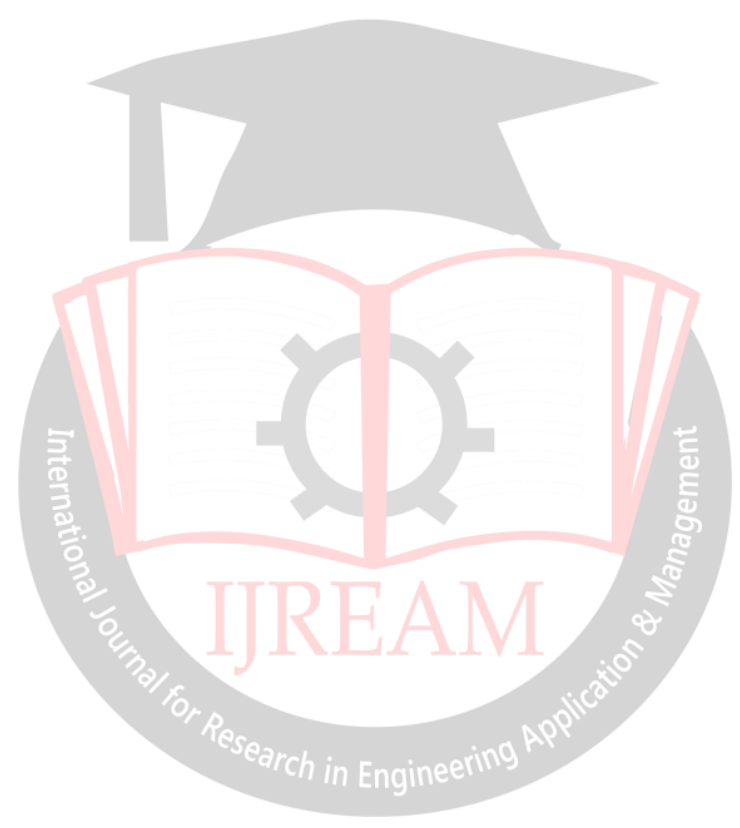

